\title{
Leveraging Local and Regional Partners to Imple- ment Hospital Closed Point of Dispensing Sites
}

\section{Daniel Baker ${ }^{1}$; Sara Paton ${ }^{2}$}

${ }^{1}$ Ohio Emergency Management Agency, Columbus, $\mathrm{OH}$

${ }^{2}$ Wright State University, Dayton, $\mathrm{OH}$

Corresponding Author: Daniel Baker, 2855 W. Dublin Granville Road, Columbus, OH 43235, (614) 799-3689, dabaker@dps.ohio.gov

Submitted December 15, 2020 Accepted April 7, 2021 Published June 21, 2021 https://doi.org/10.18061/ojph.v4i1.8078

\section{ABSTRACT}

Background: Point of dispensing (POD) sites are critical to local public health agencies (LPHA) when an emergency occurs requiring medications or vaccinations. Delivering medical countermeasures (MCM) ensures community support mechanisms and the general public are provided with a pharmaceutical intervention to limit untoward outcomes caused by the emergency. While LPHAs are required to have an MCM response plan, these plans vary based on jurisdictional size and geographical location in Ohio. This study evaluated the implementation potential of an MCM toolkit developed for LPHAs and hospitals across Ohio.

Methods: An MCM toolkit was created consisting of planning resources, training modules, sample exercises, and custom graphics. The toolkit was evaluated using Consolidated Framework for Implementation Research constructs by public health, hospital, hospital association, and emergency management professionals across Ohio by an electronic survey and phone interview. Qualitative and quantitative data were collected to determine overall implementation potential.

Results: Thirty-eight $(n=38)$ respondents with jurisdictional influence over 44 Ohio counties participated. Electronic survey results demonstrated high implementation potential for the MCM toolkit (weighted average 4.71/5.00). Phone interviews highlighted: (1) jurisdictional and regionalized planning approaches were necessary for MCM delivery, and (2) hospitals should function as closed POD sites for jurisdictional LPHAs.

Conclusion: This work demonstrates the importance of MCM response planning for LPHAs and hospitals. Implementation and planning materials for this work could be adopted into practice by LPHAs and hospitals across the United States to further assist in emergency preparedness planning.

Keywords: Public health; Emergency preparedness; Hospital preparedness; Point of dispensing; Medical countermeasures

\section{INTRODUCTION}

Local public health agencies (LPHA) play a pivotal role in the health and well-being of the communities they serve. Key pieces of public health service delivery have grown and evolved since LPHAs were formed in the late 1800 s. ${ }^{1}$ Providing services such as adult and pediatric immunizations, sexually transmitted infection testing, personal and family planning services, and breastfeeding counseling have been staples to LPHAs and the overall public health mission. ${ }^{2}$ Changing global dynamics and integration of a systems-thinking approach have greatly enhanced the roles, re- sponsibilities, and expectations of LPHAs' other core functions including emergency and disaster preparedness, epidemiological surveillance, and regional public health collaboration. ${ }^{3}$

A key component of this public health infrastructure is point of dispensing (POD) sites. The POD sites are utilized by LPHAs to rapidly dispense medical countermeasures (MCMs), medications or vaccinations, to the public before or immediately following a public health emergency. ${ }^{4}$ While the concept of POD sites is simple, the logistical need for credentialed staff, proper working environments, and community support is pronounced for LPHAs. The Cen-

(c) 2021 Daniel Baker; Sara Paton. Originally published in the Ohio Journal of Public Health (http://ojph.org) June 2021. This article is published under a Creative Commons Attribution 4.0 International License (http://creativecommons.org/licenses/by/4.0/). 
ters for Disease Control and Prevention (CDC) and the Assistant Secretary of Preparedness and Response have a joint agreement to house, maintain, and deliver MCMs to any public health jurisdiction in the United States within 12 hours of request, when available. ${ }^{4}$ The overall POD framework was then subdivided into open and closed PODs. Open PODs serve the general public, are managed by LPHA staff, and are located at large facilities (eg, arenas, schools, and community centers). ${ }^{5}$ Closed PODs are staffed and managed by individuals of a specific organization (private or public) and would only distribute MCMs to individuals who have a direct relationship to that organization (staff, immediate family of staff, and inpatient residents when applicable). ${ }^{5}$ Both open and closed PODs utilize the same planning and implementation strategy. Targeting specific populations (socially vulnerable minorities, refugees, non-English speaking populations, etc) for MCM distribution would need to be taken into account for open POD operations or as a separate initiative by the LPHA.

While the concept and planning for PODs started soon after September 2001, the first example of LPHA mobilizing PODs postSeptember 11, 2001, for a nationwide public health emergency, was in 2009 for the H1N1 influenza strain. ${ }^{3}$ H1N1 was the first post-September 11, 2001, instance where public health as a discipline was catapulted into the national spotlight for emergency response and the first opportunity POD plans were tested for a real-world emergency. ${ }^{3}$ The H1N1 outbreak of 2009 yielded several lessons learned about POD site logistics at the local government level including improved POD plans and procedures, recruitment of additional closed POD sites for LPHAs, and LPHAs integrating with hospitals and health care facilities. ${ }^{6}$ Focusing collaboration with hospitals can allow LPHA staff to leverage interventions for a large portion of the jurisdictional population through the health care system. Providing hospital staff with MCMs allows the hospital to continue critical operations during a public health emergency. The coronavirus disease 2019 (COVID-19) pandemic has shined a light on the importance and need for POD planning, training, and exercise across the public health enterprise.

While the concept of utilizing hospitals as closed POD sites is practical in theory, implementation of public health programming within the scope of a hospital is not a regularly ventured concept. Health care systems are complex and require coordination by several administrators to begin implementation of a new program. ${ }^{7}$ Even though the aforementioned challenges exist, several public health initiatives have been successfully initiated within the hospital setting in the past (eg, breastfeeding coaches, smoking cessation). ${ }^{8}$ Several organizational factors that gave rise to public health program success within the hospital setting include hospital culture and engagement, identifying where programming would fit into mission/vision, integration into organization structures, and program buy-in by administration. ${ }^{7}$

Local public health agencies are often stretched thin on staff, time, and finances to successfully implement a large-scale program within their respective field. This study focused on creating a comprehensive MCM toolkit and determining its implementation potential for LPHAs and hospitals within a jurisdiction. Additionally, the study looked at the feasibility of the MCM toolkit across urban and rural areas of Ohio. By creating a common MCM planning procedure, which the toolkit may provide, across LPHAs and hospitals, a unique opportunity exists for hospitals and LPHAs to work more in tandem and ensure continuity of health care across a community during a public health emergency.

\section{METHODS}

\section{Setting}

Local public health agencies, hospitals, regional hospital associations, and emergency management agencies (EMA) across southwest, west central, northwest, northeast, and central Ohio participated.

\section{Community, Participant Characteristics, Recruitment}

The project leader identified individuals involved in emergency preparedness activities from LPHAs, hospitals, regional hospital associations, and EMAs across 44 Ohio counties to participate. These individuals were recruited by the project leader based on professional contacts established through current and previous employment opportunities. Inclusion criteria included (1) employment in the field of public health emergency preparedness, emergency management, or health care; (2) working in the state of Ohio; and (3) being known by the project leader. No formal exclusion criteria were established.

\section{Design}

A mixed methods study was conducted using qualitative and quantitative data analysis from May 2020 to July 2020. Cross-sectional surveys were conducted electronically and by phone to identified participants from public health, emergency management, and health care to capture MCM toolkit feedback.

\section{Procedures/Program Description}

Various POD procedures, grant standards, and guidance materials from state of Ohio and CDC resources were consolidated to form the MCM toolkit. Toolkit documents were categorized into 4 primary sections: planning resources, training modules, sample exercise, and custom graphics. All documents were uploaded electronically into a cloud storage account. A document outlining all toolkit contents and potential implementation instructions accompanied the toolkit.

An electronic survey was created utilizing Microsoft Forms through Microsoft 365. The form collected reviewer information (name, contact information, jurisdiction, agency/profession, and date completed) as well as feedback on the toolkit. Toolkit document review information was ascertained based on Likert scale and open-ended questions. All open-ended questions were structured as strengths, areas of improvement, and additional com- 
ments for each section. Online survey questions were created by the project leader based on the Homeland Security Exercise and Evaluation Program (HSEEP). The HSEEP provides a common approach to exercise program design and development, evaluation, and improvement planning. ${ }^{9}$

Following the completion of the online survey, the project leader conducted a structured, 30-minute follow-up phone interview with toolkit reviewers. The project leader facilitated the discussion to determine information specific to POD implementation potential. The project leader developed follow-up questions focused on 4 Consolidated Framework for Implementation Research (CFIR) constructs: intervention characteristics, inner settings, outer settings, and process. ${ }^{10} \mathrm{~A}$ thematic analysis of all open-ended questions was conducted to determine frequency of common responses from each respondent.

\section{Measures/Outcomes}

The online survey contained quantitative and qualitative (openended) questions on MCM toolkit documents. Quantitative questions were structured on a scale from 1 to 5 where 1 represented a 'poor' rating and a 5 represented an 'excellent' rating. Open-ended questions were used to determine general strengths, areas of improvement, and general comments for each section within the online survey. All questions were to be completed to ensure uniformity in survey results and a full data comparison. Implementation potential was based on the combined weighted average (out of 5.00) from quantitative survey data. Implementation potential was categorized as either low (less than 3.00), likely (3.00-4.00), or high (greater than 4.00).

Qualitative data from the online survey were used to determine applicability of toolkit documents and identify possible implementation barriers. A thematic analysis of all open-ended questions was conducted to determine frequency of common responses from each respondent. Follow-up phone interviews provided qualitative data that identified individual agency issues to MCM planning and POD site mobilization. A thematic analysis was also conducted on phone interview responses.

\section{Statistical Analysis}

Two primary analysis techniques were used for this study, weighted average and thematic analysis. A weighted average was conducted on all quantitative survey elements using Microsoft Excel. The weighted average was conducted rather than a normal average to ensure the importance of the scale values is reflected in the results. No data points were missing from the electronic survey as all questions were deemed 'required' by the project leader. A thematic analysis was conducted on open-ended questions from both the electronic survey and the follow-up phone interviews. All thematic analyses were conducted by finding similar themes from respondents in open-ended questions and then counting frequency of those occurrences. Additionally, no data points were missing from the follow-up phone interview questions as they were also all required by the project leader.

\section{RESULTS}

A total of $38(n=38)$ surveys were completed and obtained by the project leader with Table 1 showing respondents by organization. All survey respondents comprised emergency preparedness planners for public health, hospitals, hospital associations, and EMA personnel. A 59\% participation rate can be attributed to other priorities faced by respondents for the COVID-19 pandemic response.

Table 2 provides the weighted averages (out of 5.00) of each document in each section of the toolkit. A combined weighted average for the toolkit was $4.71 / 5.00$. Because this value is greater than 4.00, this demonstrates the MCM toolkit's high implementation potential across public health, health care, and emergency management. Tables 3 and 4 show results from the qualitative thematic analysis of toolkit strengths and areas of improvement. This analysis was conducted by finding like-themes across all respondents $(n=38)$ and counting their frequency. The thematic analysis data reflect the concise nature of the toolkit as a whole by combining procedures and grant guidance materials into a single product, ability to implement, and scalability. While all toolkit materials were collected based on current state of Ohio and federal guid-

\section{Table 1. Toolkit Respondents by Organizational Area for Participation Request and Provided}

\begin{tabular}{|lll|}
\hline Organizational area & Participation request & Participation provided \\
\hline LPHA & 37 & 20 \\
Hospital & 15 & 8 \\
EMA & 7 & 6 \\
Regional hospital associations & 5 & 4 \\
\hline
\end{tabular}


Table 2. Likert Scale (out of five) Weighted Averages Results by Toolkit Review Element $(n=38)$

\begin{tabular}{|c|c|}
\hline \multicolumn{2}{|l|}{ Planning resources } \\
\hline Closed point of dispensing (POD) mobilization and response guide & 4.70 \\
\hline Memorandum of understanding (MOU) and training agreements & 4.70 \\
\hline POD staff roster template & 4.76 \\
\hline Hospital closed POD planning guide & 4.65 \\
\hline Public health cold chain management standard operating procedure (SOP) & 4.81 \\
\hline \multicolumn{2}{|l|}{ Training modules } \\
\hline POD access and functional needs training & 4.59 \\
\hline Organization chart & 4.86 \\
\hline Job action sheets & 4.84 \\
\hline Medication registration form template & 4.59 \\
\hline Vaccine registration form template & 4.68 \\
\hline POD overview training PowerPoint & 4.89 \\
\hline \multicolumn{2}{|l|}{ Sample exercise } \\
\hline Exercise evaluation guide (hospital) & 4.54 \\
\hline Exercise evaluation guide (public health) & 4.59 \\
\hline Exercise player scenarios & 4.73 \\
\hline Sample exercise - medication & 4.65 \\
\hline Sample exercise - vaccine & 4.68 \\
\hline Training evaluation (pre and post) & 4.65 \\
\hline \multicolumn{2}{|l|}{ Custom graphics } \\
\hline Registration section look and design & 4.70 \\
\hline Screening section look and design & 4.70 \\
\hline Treatment section look and design & 4.73 \\
\hline Support section look and design & 4.62 \\
\hline Exit section look and design & 4.73 \\
\hline Average (across all sections) & 4.71 \\
\hline
\end{tabular}

Table 3. Thematic Analysis of Qualitative Electronic Survey Results by Observed Theme-General Toolkit Comments-Strengths ( $\mathrm{n=38)}$

\begin{tabular}{|ll|}
\hline Theme & Frequency \\
Well organized; clear and concise information & 19 \\
Ability to implement; ease of use & 13 \\
Modular and scalable & 4 \\
Customizable & 3 \\
Graphics & 2 \\
\hline
\end{tabular}

Table 4. Thematic Analysis of Qualitative Electronic Survey Results by Observed Theme-General Toolkit Comments-Areas of Improvement $(\mathbf{n}=38)$

\begin{tabular}{|lc|}
\hline Theme & Frequency \\
None & 20 \\
Spelling and/or grammatical revisions & 4 \\
Customization (graphics, PowerPoints) & 3 \\
Exercise scenario modification & 3 \\
Document length (longer than preferred) & 2 \\
\hline
\end{tabular}


ance, few respondents felt the toolkit was customizable enough to fit the needs of their organization.

During the follow-up interview, reviewers identified the POD toolkit as an item that will be integrated into current POD planning activities for internal and external partners. Comments supporting this finding included

"This toolkit will be utilized by my entire hospital system. Everything laid out provides enough information for our hospitals to quickly become a closed POD site. Additionally, we can easily work with the health department to get up to speed on what is needed to be an official POD site for them." (hospital planner);

"We can add to what is already in place for POD planning with the addition of this toolkit. Everything is laid out very nicely and can be very easy for patients who would be moving through a POD using this model." (LPHA planner); and

"The toolkit really brings home the point of engaging public/ private partnerships. We have never had to do so much with the health department until the COVID-19 pandemic - we now realize how much more we have to rely on the private sector," (EMA director).

These comments highlighted both the implementation potential for the toolkit and the need to engage public-private partnerships. Emergency management directors discussed the complexity of public health emergencies and the need to have a coordinated response with the private sector. Both hospital and LPHA planners cited the ease of implementation for new or existing MCM plans. While the concept of establishing a closed POD within the hospital is new, both LPHAs and hospitals agreed that it is not only attainable but that it would be beneficial to each agency during an emergency.

When asked about potential internal implementation barriers, respondents unanimously agreed that coordination of POD plans between LPHAs and hospitals should occur at the jurisdictional and regional levels. Comments that support this finding included

"Regional and local planning needs to happen with the POD toolkit - even without. Our plans are all different and we each do things a little different in each county. This should be done with all of our plans, honestly," (LPHA planner); and

"Regional planning allows hospitals and LPHA to have better handle on resources and move resources around as needed - it provides a more coordinated response. That is critically important for a POD and movement of life saving resources. We need to have better coordination in our regions," (hospital association coordinator).

The comments cited above underscore the importance of jurisdictional and regional planning. While these comments only capture an LPHA planner and hospital association coordinator, the sentiments were shared by a majority of respondents. Ensuring prepar- edness planning efforts encompass all relevant partners is paramount when a real-world incident occurs.

\section{DISCUSSION}

This work provides a foundational baseline for other practitioners and academicians to better understand an integral piece of public health practice and public health infrastructure. The significance of public health emergency preparedness has never been more understood given the COVID-19 pandemic. The MCM toolkit provides a mechanism for practitioners in public health, health care, and emergency management to implement and evaluate their overall preparedness planning for a public health emergency.

Findings from follow-up phone interviews with LPHAs and hospitals suggest that hospitals can be leveraged by LPHAs to serve as a closed POD for the community. As evidenced by the COVID-19 pandemic, LPHAs and hospitals are both tasked with a myriad of responsibilities to safeguard the public's health. The introduction of hospital closed POD sites not only expedites the MCM distribution process within a jurisdiction, but it will allow for better continuity of operations for both entities. This concept reinforces the notion of utilizing systems-based approaches to complete public health services.11,12 Funding for this initiative would be a joint partnership between the LPHA's and hospital's respective general revenue funds or preparedness grants.

Systems thinking can also include leveraging public-private partnerships. Leveraging public-private partnerships was cited by toolkit reviewers in follow-up interviews as a need for LPHAs moving forward. DeSalvo and colleagues point out improved partnerships throughout the community are necessary to sustain public health services. ${ }^{13}$ The Federal Emergency Management Agency's (FEMA) revised emergency support function (ESF) 14 also outlines a new approach for public-private partnership integrating into emergency response for local, state, and federal emergency management. ${ }^{14}$ Revised ESF 14 can assist LPHAs to recruit hospitals as closed POD sites, as well as other private organizations in the future, by providing a planning framework for local EMA directors to coordinate the process.

Findings from the electronic survey (Table 3) suggested that the MCM toolkit is modular, scalable, and can easily be implemented. While establishing hospital closed POD sites was shown to be a vital component for LPHAs in the follow-up phone interviews, the widespread dissemination of an MCM toolkit must show itself to be scalable, modular, and customizable to best fit the needs of a jurisdiction. The prepared MCM toolkit provided a method to incorporate these differences by integrating HSEEP principles. The HSEEP has been used as a methodology to conduct planning, training, and exercises as modular and scalable units. ${ }^{9}$ This method has been shown to be the most effective for adult learning and applicability to various sized jurisdictions. ${ }^{9,15}$ The HSEEP provides a means to underscore general lecture style training that is capped off with a discussion-based or action-based exercise. Because of 
the various types of emergency response partners within a community (public health, hospitals, emergency management, hospital associations, law enforcement, etc), scalability is needed to encompass the strengths of different sized response agencies. This concept is also true for understanding the roles and responsibilities of these agencies.

The CDC's public health emergency preparedness capabilities have been utilized to demonstrate the applicability of concepts to both urban and rural jurisdictions and were shown to be an effective metric for preparedness performance. ${ }^{16}$ Counties across Ohio vary in size from over 1 million individuals in urban areas to less than 14 thousand in rural areas. ${ }^{17}$ Each jurisdiction is also vastly different in resources, political structure, and staffing capability. The respondents for this study encompassed both urban and rural counties across Ohio. Based on the results in Table 3 and the follow-up phone interview quotes, this study demonstrated that the MCM toolkit allows for scalability and customization by LPHAs and hospitals regardless of jurisdictional size or geographical location through the state of Ohio. Additionally, the modular nature of the materials allows for LPHAs to conduct planning, training, and exercise that fits into a cycle that best fits their needs. Scalability is imperative because it allows individuals to utilize the toolkit to the fullest extent necessary for their respective jurisdiction. This ensures that the basic principles of MCM response are maintained from the toolkit while allowing for flexibility during implementation.

Finally, this work provided evidence to highlight the importance of regional planning efforts within the state of Ohio. As evidenced by the implementation barriers in the results, regionalized planning is a concept that had not been well received by local jurisdictions in the past and can be seen as loss of control or a means of political conflict. Planners within public health and hospitals often use their social networks to collaborate on different projects, deliverables, and grant requirements within regions. Individual jurisdictions may implement a common planning structure (eg, MCM toolkit) and the structure is then used with LPHAs and hospitals within their respective jurisdiction. This conceptualization could then graduate to being adopted by other counties within a region. Progression from individual jurisdictional planning to a regionalized approach could be a possible framework to be considered by LPHA and hospital planners to address concerns outlined in toolkit implementation barriers.

Harris and Clements highlighted the need for information exchange between public health practitioners through their social networks. ${ }^{18}$ Loss of experience based on personnel turnover within public health increases the need for these social networks and other unique approaches to ensure public health services are maintained at the local level.3,13,18 Sharing of resources and personnel, as well as the ability to save on costs and general overhead, were cited as major benefits to regional planning. ${ }^{3}$ This concept was also shown to provide a foundational approach to allow POD planning for all jurisdictions to ensure continuity among all
LPHAs and hospitals. This allows for a unified approach between LPHAs and hospitals to expedite the MCM distribution process to residents within jurisdictions.

A limitation to this study was generalization of results. Because study participants were obtained through a convenience sample, external validity may not account for the larger public health system.

\section{PUBLIC HEALTH IMPLICATIONS}

The implication of MCM toolkit dissemination would substantially benefit the public health community across Ohio and the United States. Toolkit reviewers determined that the scalability and implementation potential is applicable for both rural and urban areas. While not explicitly reviewed for this study, exurban and suburban areas would need to be considered as part of the primary jurisdiction. The MCM toolkit has implementation potential for jurisdictions across Ohio and the United States. Additionally, the use of regionalized planning can be utilized by local-level partners for other planning considerations outside of MCM response (eg, epidemiological outbreak, environmental health emergency, etc). Leveraging regional planning efforts streamlines processes and allows for a shared area of responsibility among similar partner agencies.

Revised ESF 14 guidance produced by FEMA encourages the coordination and utilization of private partners when responding to an emergency. While hospitals are the first step toward leveraging the public-private partnership for MCM distribution in communities, revised ESF 14 will better equip local EMA and emergency operation centers to identify additional private partnerships by designating specific planning considerations for partnership integration. Private pharmacies, grocery stores, and biotech companies have been leveraged and engaged during the COVID-19 response for testing purposes. The same principle of resource identification and integration may be utilized by LPHAs and local EMAs to cultivate additional public-private partnerships.

\section{ACKNOWLEDGMENTS}

The project leader would like to thank the many professionals across Ohio who provided their input and time into this research. This support demonstrates their commitment to establishing more evidence-based public health practices in Ohio. The project leader would also like to thank Ishmael Hoare, PhD, University of South Florida for his support.

\section{REFERENCES}

1. State of Ohio. Ohio Revised Code $§ 3709.01$ - Health districts. Ohio Revised Code $\$ 3709.01$ - Health districts. Ohio Revised Code $\$ 3709.01$ Health districts. Published 2013.

2. Sellers K, Leider JP, Gould E, et al. The state of the US governmental public health workforce, 2014-2017. Am J Public Health. 2019;109 (5):674-680. https://doi.org/10.2105/AJPH.2019.305011

3. Lurie N, Wasserman J, Nelson CD. Public health preparedness: evolution or revolution? Health Aff. 2006;25(4):935-945. https://doi.org/10.1377/hlthaff.25.4.935 
4. Centers for Disease Control and Prevention. Division of Strategic National Stockpile Program Review. 2012.

https://www.cdc.gov/cpr/science/documents/dsns_program_review_ workgroup_report_final2.pdf

5. Centers for Disease Control and Prevention. Health Care Closed Points of Dispensing. Published 2018.

https://www.cdc.gov/cpr/readiness/healthcare/closedPODtoolkit.htm

6. Department of Homeland Security. 2009 H1N1 After Action Report; 2010. https://www.hsdl.org/?view\&did=783079

7. Sligo J, Gauld R, Roberts V, Villa L. A literature review for large-scale health information system project planning, implementation and evaluation. Int J Med Inform. 2017;97(2017):86-97.

https://doi.org/10.1016/j.ijmedinf.2016.09.007

8. Vanderkruik R, McPherson ME. A contextual factors framework to inform implementation and evaluation of public health initiatives. $\mathrm{Am} \mathrm{J}$ Eval. 2017;38(3):348-359. https://doi.org/10.1177/1098214016670029

9. Federal Emergency Management Agency. Homeland Security Exercise and Evaluation Program.

10. Consolidated Framework for Implementation Research. What is the Consolidated Framework for Implementation Research?Published 2019.

https://cfirguide.org/

11. Institute of Medicine. The Future of Public Health. The National Academies Press. 1988.

https://doi.org/10.17226/1091

12. Pickett G. The future of public health. J Public Health Policy. 1989;10

(3):397.

https://doi.org/10.2307/3342799

13. DeSalvo KB, Claire Wang Y, Harris A, Auerbach J, Koo D, O'Carroll P. Public health 3.0: A call to action for public health to meet the challenges of the 21st century. Prev Chronic Dis. 2017;14(9):1-9.

https://doi.org/10.5888/pcd14.170017

14. Federal Emergency Management Agency. Emergency Support Function \# 14 - Cross-Sector Business and Infrastructure. 2019;(October):1-15. https://www.fema.gov/media-library-data/1572358162675d2c7af34a5b5063e582ae1798b038351/ESF14AnnexFINAL508 C_20191028.pdf

15. Hites LS, Granillo BS, Garrison ER, et al. Emergency preparedness training of tribal Community Health Representatives. J Immigr Minor Heal. 2012;14(2):323-329. https://doi.org/10.1007/s10903-011-9438-9

16. Hunter JC, Yang JE, Crawley AW, Biesiadecki L, Aragón TJ. Public health response systems in-action: Learning from local health departments' experiences with acute and emergency incidents. PLoS One. 2013;8 (11). https://doi.org/10.1371/journal.pone.0079457

17. United State Census Bureau. Population and Housing Unit Estimates Datasets. Published 2018. https://www.census.gov/programs-surveys/popest/data/datasets.html

18. Harris JK, Clements B. Using social network analysis to understand Missouri's system of public health emergency planners. Public Health Rep. 2007;122(4):488-498.

https://doi.org/10.1177/003335490712200410 


\section{APPENDIX. Electronic Survey Template POD/MCM Online Survey}

1. What organization do you represent?

Local public health agency

Hospital

Regional hospital association (eg, HCNO, GDAHA, etc)

Local emergency management agency

2. What is your name?

3. Name of your organization's county of residence.

4. What is your email address?

5. What is the best phone number to reach you for follow-up questions?

6. When reviewing the PLANNING RESOURCES folder, please provide your feedback to each of the following items. One (1) reflect a rating of "poor" while a five (5) reflects a rating of "great."

Closed Point of Dispensing (POD) Mobilization and Response Guide

1 (Poor) $2 \quad 3 \quad 4 \quad 5$ (Great)

MOU and Training Agreements

1 (Poor) 2345 (Great)

POD Staff Roster Template

1 (Poor) $2 \quad 345$ (Great)

Hospital Closed POD Planning Guide

1 (Poor) $2 \quad 345$ (Great)

Public Health Cold Chain Management SOP

1 (Poor) $2 \quad 3 \quad 45$ (Great)

7. Please describe notable strengths for the PLANNING RESOURCES materials.

8. Please describe notable areas of improvement for the PLANNING RESOURCES materials.

9. Please include any additional comments for the PLANNING RESOURCES materials.

10. When reviewing the TRAINING MODULES folder, please provide your feedback to each of the following items. One (1) reflect a rating of "poor" while a five (5) reflects a rating of "great."

POD Access and Functional Needs Training

1 (Poor) $23 \quad 3 \quad 4 \quad 5$ (Great)

Organization Chart

1 (Poor) $22 \quad 3 \quad 4 \quad 5$ (Great)

Job Action Sheets

1 (Poor) $23 \quad 3 \quad 5$ (Great)

Medication Registration Form Template

1 (Poor) $23 \quad 345$ (Great)

Vaccine Registration Form Template

1 (Poor) $23 \quad 345$ (Great)

POD Overview Training PowerPoint

1 (Poor) $23 \quad 345$ (Great)

11. Please describe notable strengths for the TRAINING MODULES materials.

12. Please describe notable areas of improvement for the TRAINING MODULES materials.

13. Please include any additional comments for the TRAINING MODULES materials.

14. When reviewing the SAMPLE EXERCISE folder, please provide your feedback to each of the following items. One (1) reflect a rating of "poor" while a five (5) reflects a rating of "great."

Exercise Evaluation Guide (Hospital)

1 (Poor) 22345 (Great)

Exercise Evaluation Guide (Public Health)

1 (Poor) 22345 (Great)

Exercise Player Scenarios
1 (Poor) 2345 (Great)

Sample Exercise - Medication

1 (Poor) $2 \quad 3 \quad 4 \quad 5$ (Great)

Sample Exercise - Vaccine

1 (Poor) 2345 (Great)

Training Evaluation (Pre and Post)

1 (Poor) $2 \begin{array}{llll}3 & 4 & 5 \text { (Great) }\end{array}$

15. Please describe notable strengths for the SAMPLE EXERCISE materials.

16. Please describe notable areas of improvement for the SAMPLE EXERCISE materials.

17. Please include any additional comments for the SAMPLE EXERCISE materials.

18. When reviewing the CUSTOM GRAPHICS folder, please provide your feedback to each of the following items. One (1) reflect a rating of "poor" while a five (5) reflects a rating of "great."

Registration Section Look and Design

1 (Poor) 2345 (Great)

Screening Section Look and Design

1 (Poor) 23345 (Great)

Treatment Section Look and Design

1 (Poor) 2345 (Great)

Support Section Look and Design

1 (Poor) $2 \quad 3 \quad 4 \quad 5$ (Great)

Exit Section Look and Design

1 (Poor) $23 \quad 3 \quad 4 \quad 5$ (Great)

19. Please describe notable strengths for the CUSTOM GRAPHICS materials.

20. Please describe notable areas of improvement for the CUSTOM GRAPHICS materials.

21. Please include any additional comments for the CUSTOM GRAPHICS materials.

22. Given the OVERALL TOOLKIT, please describe notable strengths for all the materials presented to you.

23. Given the OVERALL TOOLKIT, please describe notable areas of improvement for all the materials presented to you. 